\title{
Defect detection in textured materials using Gabor filters
}

\author{
Ajay Kumar, Grantham Pang \\ Industrial Automation Research Laboratory \\ Dept. of Elec. \& Electronic Engineering, \\ The University of Hong Kong, \\ Pokfulam Road, Hong Kong. \\ Tel:(852)-2857-8492 Fax:(852)-2559-8738 \\ Email: gpang@hkueee.hku.hk
}

\begin{abstract}
Vision-based inspection of industrial materials such as textile webs, paper, or wood requires the development of defect segmentation techniques based on texture analysis. In this work, a multi-channel filtering technique that imitates the early human vision process is applied to images captured on-line. This new approach uses Bernoulli's rule of combination for integrating images from different channels. Physical image size and yarn impurities are used as key parameters for tuning the sensitivity of the proposed algorithm. Several real fabric samples along with the result of segmented defects are presented. The results achieved show that the developed algorithm is robust, scalable and computationally efficient for detection of local defects in textured materials.
\end{abstract}

\footnotetext{
I. Introduction

Industrial inspection problems are typically highly complex and require integrated solutions that can be executed in real time. Some of the most challenging industrial inspection problems deal with textured materials such as textile web, paper and wood. It should be noted that the inspection problems encountered in uniforms webs become texture analysis problems at microscopic levels. The detection of local fabric defects is one of the most intriguing
}

and difficult problems in computer vision and has received much attention over the years $[1,2]$.

Differences in the mean gray level or in color in small neighborhoods alone are not always sufficient for defect detection. Rather, one has to rely on differences in the spatial arrangement of gray level values in the neighboring pixels. Defect segmentation involves identification of regions with uniform texture in a given image. Appropriate measures of texture are needed in order to decide whether a given region has uniform texture [3]. A region in an image has a constant texture if a set of local statistics or other local properties of texture are constant, slowly varying, or approximately periodic [4].

Different approaches based on feature extraction in the spatial domain have been proposed for the detection of local defects on textile webs. An adapted wavelet basis has high sensitivity to abrupt changes in the texture structure caused by defects. Jasper et al. [5] demonstrate how adaptive wavelet basis can be used to locate defects in fabric. Although Jasper et al. [5] did not rely on capability of Gabor filters to detect local fabric defects, Escofet et al. [6] has demonstrated this in their work. 
The main contributions of this paper are summarized below.

1. Bernoulli's rule of combination is proposed to integrate information from different channels. This approach offers high detection rate and low false alarm.

2. Introduction of 'variable sensitivity' improves the performance of the developed algorithm in the presence of yarn impurities and for large (physical) size images.

3. High sensitivity and small convolution mask makes this algorithm computationally economic, and hence increases the on-line performance.

4. Supervised defect detection using optimal Gabor filters [7] is also presented.

\section{Gabor filter}

In spatial domain a Gabor function is modulation product of complex exponential and a Gaussian envelope of arbitary duration (figure 1). In frequency it is seen as a shifted Gaussian. In general, a two-dimensional Gabor function is expressed as $[8,9]$ :

$$
\begin{aligned}
\psi\left(x, y, \omega_{i}, \varphi_{\mathrm{j}}\right)= & \exp \left(-\pi\left[\left(\frac{\mathrm{x}}{\sigma_{\mathrm{x}}}\right)^{2}+\left(\frac{\mathrm{y}}{\sigma_{\mathrm{y}}}\right)^{2}\right]\right) \\
& \exp \left(\mathrm{j} \omega_{\mathrm{i}}\left(\mathrm{x} \cos \varphi_{\mathrm{j}}+\mathrm{y} \sin \varphi_{\mathrm{j}}\right)\right)
\end{aligned}
$$

An important property of Gabor filters is that they achieve maximum possible joint localization, or resolution in both spatial and spatial-frequency domains [10]. One of their drawbacks is that they are non-orthogonal, but complete for representation of visual information. The Gabor filter owes its feature extraction capability to the possibility of tuning the orientation of its frequency selectivity. The frequency (octave) and orientation (radian) bandwidths of the Gabor filter is given by [11],

$$
\Theta_{r_{i}}=\log _{2}\left(\frac{\omega_{i} \sigma+\sqrt{2 \ln 2}}{\omega_{i} \sigma-\sqrt{2 \ln 2}}\right) \quad \Omega_{\varphi_{i}}=2 \tan ^{-1}\left(\frac{\sqrt{2 \ln 2}}{\omega_{i}}\right)
$$

Supervised defect detection in textured materials can be performed with a Gabor filter optimized with respect to feature separation from a known defect. As proposed by Dunn and Higgins [7], the optimal Gabor filters can be constructed from the filters with center frequency yielding minimum classification error. An example of optimal Gabor filter designed to detect fabric defects is shown in figure 2 . The acquired fabric image is filtered with this optimal Gabor filter and defect can be segmented by thresholding (figure 2.d). In order to cope up with large variety of defects, unsupervised defect detection is more suitable for web inspection and is detailed in following sections.

\section{Feature extraction}

Image features are extracted by power spectrum sampling of acquired image. For this purpose log-polar spectrum of acquired image is divided into four scales $\left(\omega_{1}, \omega_{2}, \omega_{3}\right.$ and $\left.\omega_{4}\right)$, separated by an octave. Sampling points at each of these four frequencies is rotated in steps of $45^{\circ}$ to cover entire spectrum of image. Gabor filters placed at each of these 16 sampling points are implemented as convolution masks of smaller dimensions, typically $5 \times 5$ or $7 \times 7$. A set of 16 filtered image from these Gabor filters form feature vector for defect detection.

Low frequency features of acquired image are used for feature enhancement. Subsequent repitition of subsampling and convolution with low pass filter generates a set of images with varying degree of resolution (multiresolution pyramid). The images generated from this four stage pyramid decomposition are used to normalize 16 Gabor filtered images. Thus each image from image pyramid normalizes four Gabor filtered images in each scale. 


\section{Defect detection}

A defect free image of sample under test is used as reference (figure 4). With this reference image, a set of 16 feature images (as discussed in section 4) are generated. Mean $\left(\mu_{i j}\right)$ and standard deviations $\left(\sigma_{i j}\right)$ from each of these sixteen images (16 pairs) form the basis of locating defect in sample under test. Next, the 16 feature images from sample under test are transformed to corresponding decision images as follows:

$$
D_{i j}(x, y)= \begin{cases}F_{i j}(x, y) & \left|F_{i j}(x, y)-\mu_{i j}\right| \geq\left|\zeta \sigma_{i j}\right| \\ 0 & \text { Otherwise }\end{cases}
$$

The parameter $\zeta$ determines the sensitivity of this algorithm. This largely depends on image acquisition conditions and impurities present in sample under inspection.

\section{Image fusion}

The task of image fusion module is to combine all pixels with high probability of being defect (from 16 images) into a single image. This is achieved in three stages. In the first stage, four images from each of the four scales are fused into single image using Bernoulli's rule $[12,13]$ of combination.

$$
\begin{aligned}
T_{i}(\mathrm{x}, \mathrm{y})= & \sum_{\mathrm{j}=1}^{4} R_{i j}(\mathrm{x}, \mathrm{y})- \\
& \left\{\begin{array}{l}
R_{i 1}(\mathrm{x}, \mathrm{y}) R_{i 2}(\mathrm{x}, \mathrm{y})+R_{i 2}(\mathrm{x}, \mathrm{y}) R_{i 3}(\mathrm{x}, \mathrm{y}) \\
+R_{i 3}(\mathrm{x}, \mathrm{y}) R_{i 4}(\mathrm{x}, \mathrm{y})+R_{i 4}(\mathrm{x}, \mathrm{y}) R_{i l}(\mathrm{x}, \mathrm{y}) \\
+R_{i 4}(\mathrm{x}, \mathrm{y}) R_{i 2}(\mathrm{x}, \mathrm{y})+R_{i 3}(\mathrm{x}, \mathrm{y}) R_{i I}(\mathrm{x}, \mathrm{y})
\end{array}\right\}
\end{aligned}
$$

Where $R_{i j}(x, y)$ is obtained from $D_{i j}(x, y)$ by normalizing its pixel values in 0 1 range. In the second stage, the false alarms from these four images $\left(T_{i}(x, y)\right)$ are further reduced by taking geometric mean of images at adjacent scales. In the last stage, the arithmetic mean of these three images generates a fused image output.
Finally, the thresholding of this fused image produces the binary image of defects in sample under inspection. The thresholding value depends on texture of material and imaging system, and is obtained by calibrating the system at the beginning of inspection.

\section{Results}

The imaging subsystem consists of a 385 x 287 CMOS array connected to parallel port of a PC. Twill and plain weave fabric samples with most commonly occurring defects were gathered from textile factory and used to evaluate this algorithm. Some of these results are reproduced here. All of these images in figure 6 cover $10 \mathrm{~cm}$ width and $7.5 \mathrm{~cm}$ height of the fabric, moving with the velocity of 30 $\mathrm{cm} / \mathrm{sec}$. Due to an increase in area of fabric per frame, we have increased the sensitivity of the algorithm $(\zeta=1)$. The results achieved confirm that this algorithm is robust, scalable, and offers high detection rate.

\section{Conclusions}

In this paper an algorithm based on multichannel filtering has been successfully used for defect detection in textile web. The significant gain in performance are attributed to (a) usage of Bernoulli's principle in image fusion, (b) higher sensitivity in conjunction with low spatial sampling and, (c) lower sensitivity in presence of yarn impurities. Furthermore, considerable computational saving has been achieved with the use $7 \times 7$ masks. This algorithm is also well suited for inspection of any other textured materials such as timber or plastic.

\section{References}

1. C.H.Chan, H.Liu, T.Kwan, G. Pang, "Automated technology for fabric inspection system," Conference on applications of automation science and 
technology, City University of Hong Kong, Nov.24-26, 1998.

2. H.S. Liu, C.H.Chan, T. Kwan, G.Pang, "Neural-Computing in fabrics inspection system," Int. Symp. on Soft Computing in Engineering applications, Athens, Greece, June 22-25, 1998.

3. A.K. Jain and F. Farrokhnia, "Unsupervised texture segmentation using Gabor filters," Pattern Recognit., vol. 24, pp. 1167-1169, 1991.

4. J. Sklansky, "Image segmentation and feature extraction," IEEE Trans. Syst. Man, Cybern., vol. 8, pp. 237-247, 1978.

5. Warren J. Jasper, Stephen J. Garnier and Harsh Potlapalli, "Texture characterization and defect detection using adaptive wavelets," Opt. Eng., vol. 35, no. 11, pp. 3140-3149, Nov. 1996.

6. J. Escofet, R. Navarro, M.S. Millan, and J. Pladelloreans, "Detection of local defects in textiles webs using Gabor filters," Opt. Eng., vol. 37, no. 8, pp. 2297-2307, Aug. 1998.

7. D.F. Dunn and W.E. Higgins, "Optimal Gabor filters for texture segmentation," IEEE Trans. Image.Processing, vol. 7, pp. 947-964, 1995.

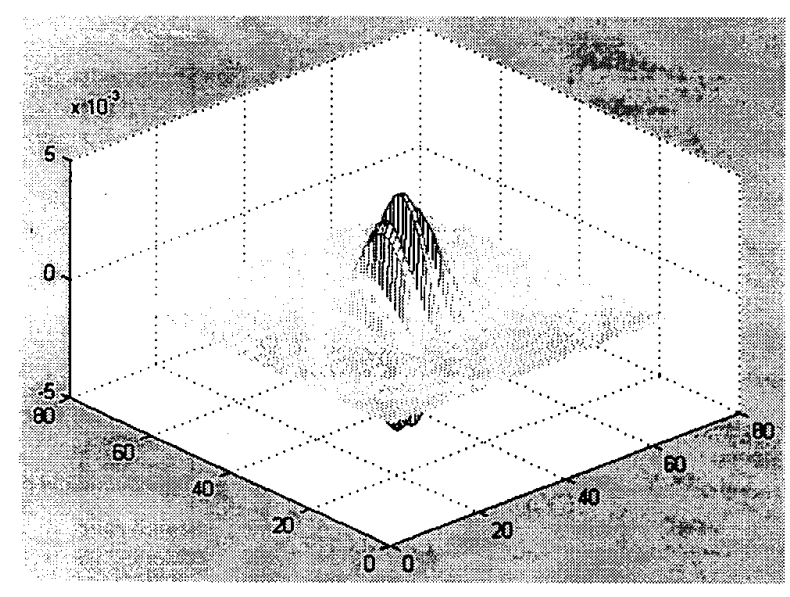

8. D. Gabor, "Theory of Communication," $J$. Inst. Elect. Eng., vol. 93, pp. 429-457, 1946.

9. Z.Q. Liu, R.M. Rangayyan, and C.B. Frank, "Analysis directional features in images using Gabor filters," in Proc. $3^{\text {rd }}$ Annual IEEE Symp. on Computer-Based Medical Systems, pp. 68-74, 1990.

10. J. Daugman, "Uncertainty relation for resolution in space, spatial frequency, and orientation optimized by two-dimensional visual cortical filters," J. Opt. Soc. Amer., vol. 2, no. 7, pp. 1160-1169, 1985.

11. J. Portilla, R. Navarro, O. Nestares and A. Tabernero, "Texture synthesis-by-analysis method based on a multiscale early-vision model," Opt. Eng., vol. 35, no. 8, pp. 2403-2417, Aug. 1998.

12. M. Abdulghafour, J. Goddard, and M. A. Abidi, "Non-Deterministic Approaches in Data Fusion - A Review," SPIE Conf. on Sensor Fusion, vol. 1393, Boston, MA, pp. 596-610, Nov. 1990.

13. H. Sari-Sarraf and J. S. Goddard, "Vision systems for on-loom fabric inspection," in IEEE Annual Conf. on Textile, Fiber and Film Industry, pp. 1-11, 1998.

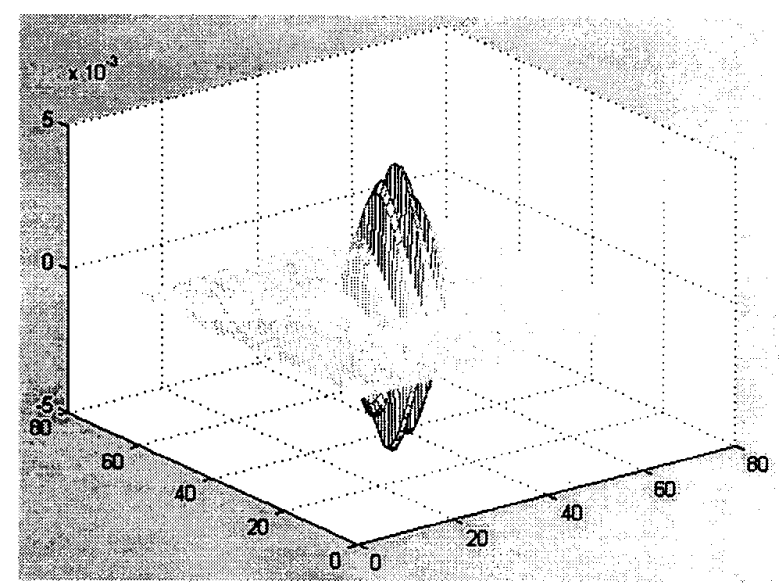

Figure 1: Perspective view of Real and Imaginary components of a Gabor filter in spatial domain. 


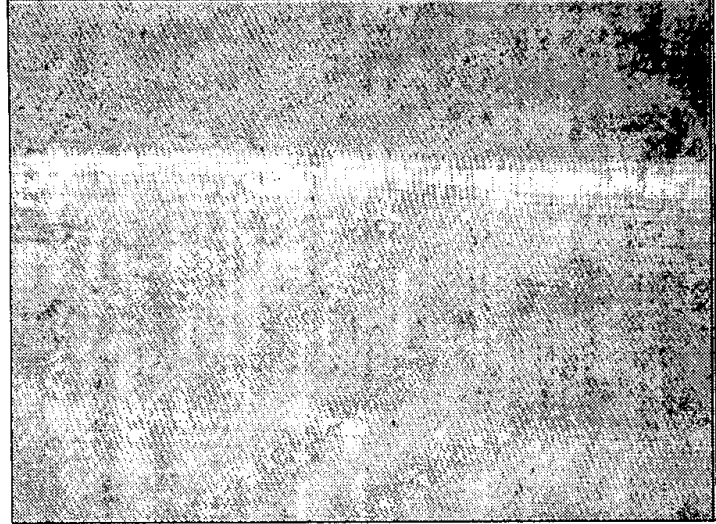

(a)

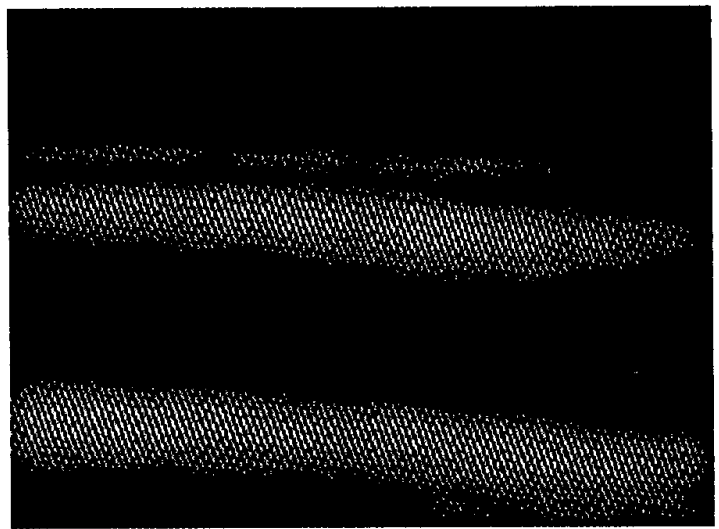

(c)

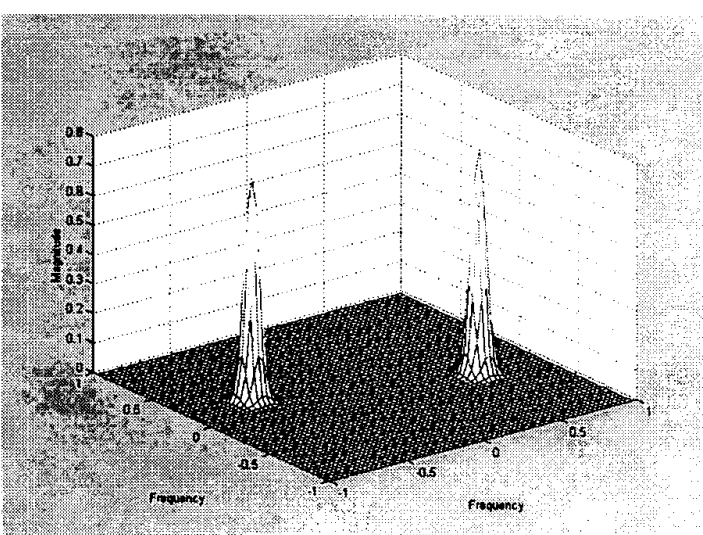

(b)

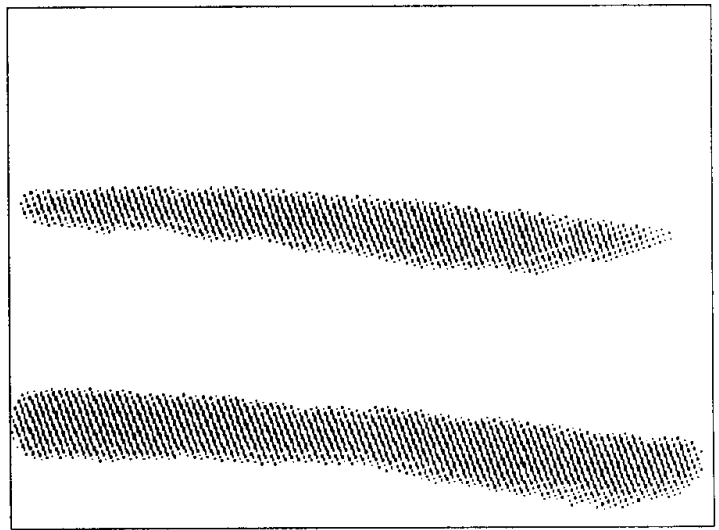

(d)

Figure 2: Supervised defect detection using optimal Gabor filters: (a) Fabric sample with defect, (b) magnitude frequency response for optimal Gabor filter, (c) filtered image and (d) segmented defect.

\begin{tabular}{|c|c|}
\hline On-line fabric images & Gabor filter Bank \\
\hline Image Thresholding & Feature enhancement \\
\hline Segmented defects & Figure 3: Block diagram of on-line defect detection algorithm. \\
\hline
\end{tabular}




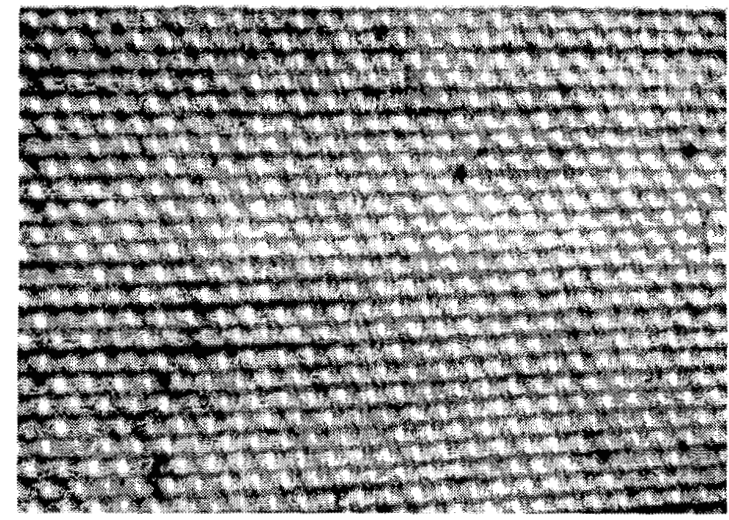

(a)

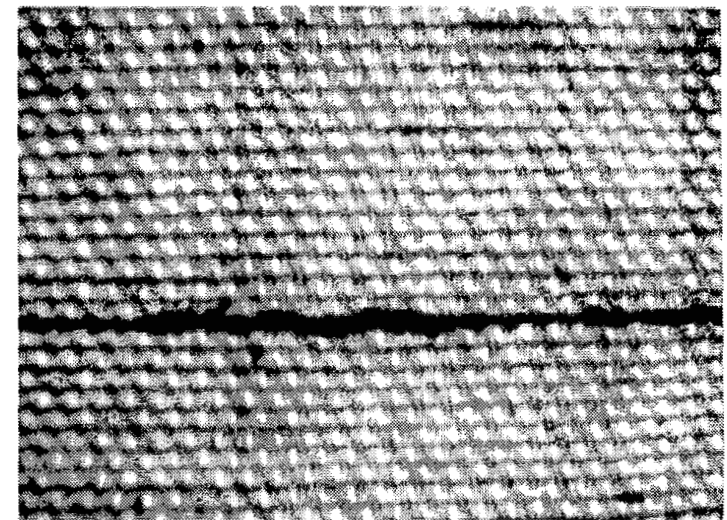

(b)

Figure 4: Fabric sample for test (a) without defects, (b) with defects

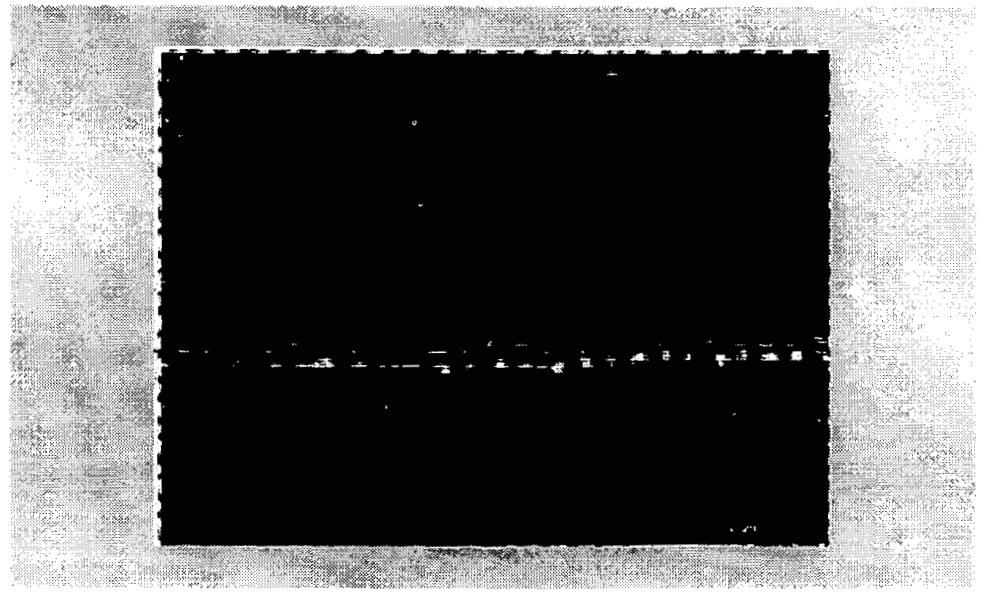

Figure 5: Segmented defect for fabric sample shown in figure 3. 


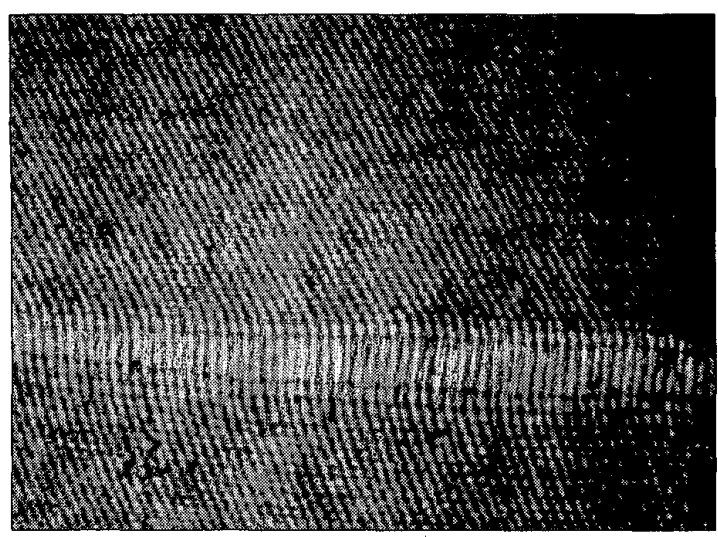

(a)

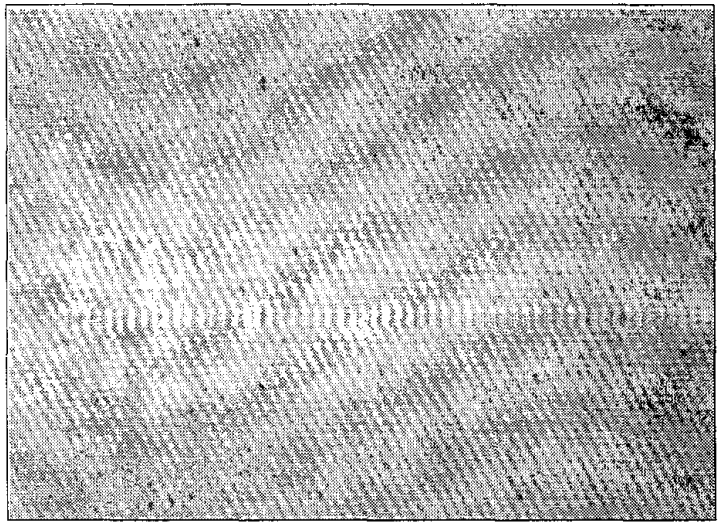

(c)

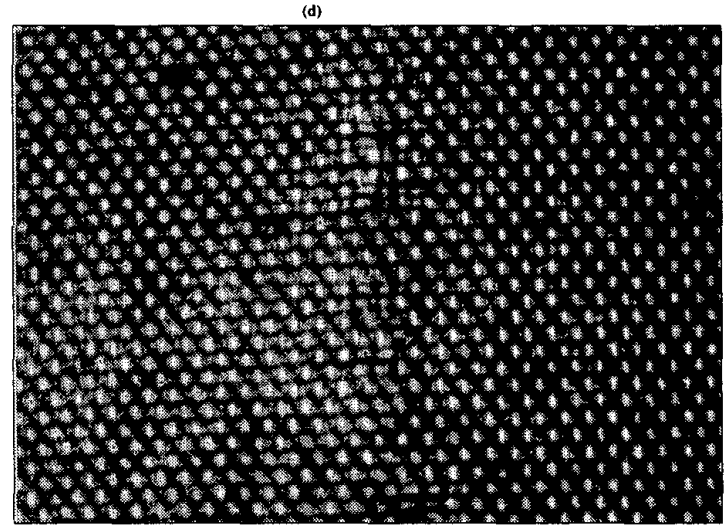

(e)

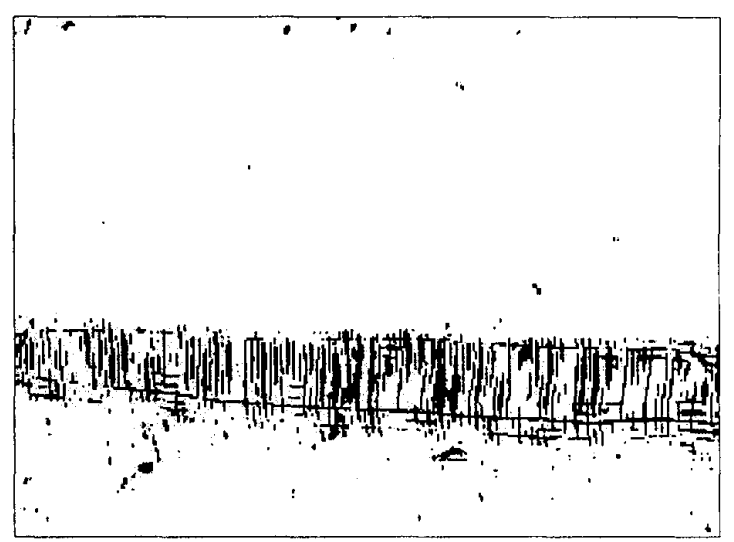

(b)

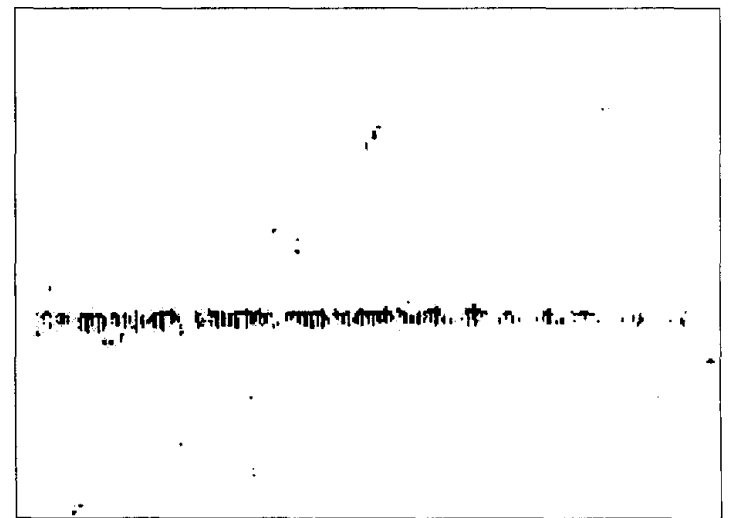

(d)

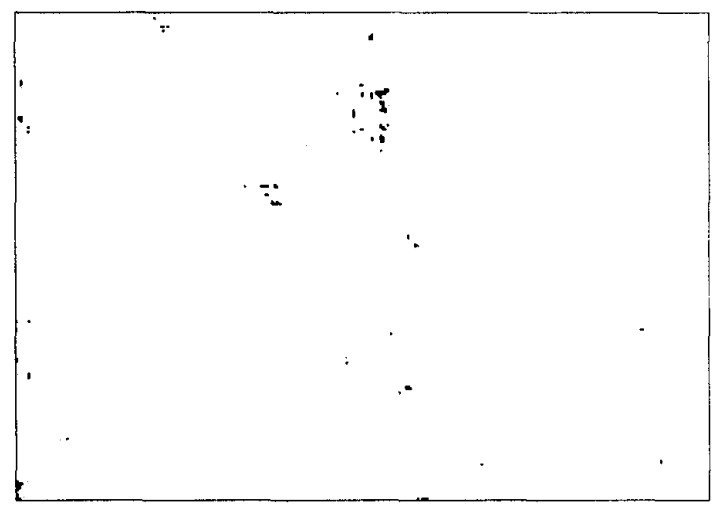

(f)

Figure 6: Various defective fabric test samples (a),(c),(e), and binarized segmented defect (b),(d),(f). 